\title{
Avaliação dos fatores preditivos de invasão neoplásica do complexo areolomamilar em pacientes com câncer de mama
}

\author{
Evaluation of the predictive factors involved in the neoplastic invasion of the nipple-areolar complex in \\ patients with breast cancer
}

Ana Thereza da Cunha Uchôa Camacho'; Alexandre Rolim da Paz; Paula Carvalho de Abreu-e-Lima ${ }^{3}$; Maria do Carmo Carvalho de Abreu-e-Lima ${ }^{4}$

unitermos
Mama
Carcinoma
Mamilos

\section{resumo}

Introdução: A escolha pela mastectomia com preservação do complexo areolomamilar (CAM), de melhor resultado estético, depende da ausência de envolvimento neoplásico do CAM. Objetivo: Identificar os fatores preditivos de invasão neoplásica do CAM. Método: Foi realizado estudo analítico, retrospectivo, clínico-patológico de 48 peças de mastectomia de pacientes portadoras de carcinoma da mama, atendidas no Hospital de Câncer da Paraíba - Fundação Napoleão Laureano, durante o período de abril de 2007 a janeiro de 2008, com idade média de 52,69 \pm 10,72 anos. O CAM foi seccionado separadamente mediante cortes perpendiculares e paralelos a partir da papila, estendendo-se a $2 \mathrm{~cm}$ de profundidade do tecido retroareolar (com o intuito de incluir seios lactíferos e ductos coletores e segmentares), seguidos de cortes transversais até a base. Resultados: Identificou-se frequência de envolvimento do CAM por carcinoma invasivo em $14,58 \%$ dos casos. Pacientes jovens (média 42,3 $\pm 4,7$ anos), distância do tumor a mamilo/aréola $\leq 2 \mathrm{~cm}$, tamanho da aréola $\leq 3 \mathrm{~cm}$, tipo histológico (carcinoma ductal invasivo associado a carcinoma ductal in situ padrão comedônico), status dos linfonodos axilares (sobretudo com metástases em 10 ou mais linfonodos), presença de invasão angiolinfática ou perineural e existência de infiltrado inflamatório dos estromas intratumoral e peritumoral foram associados a maior risco de envolvimento do CAM $(p<0,05)$. O menor diâmetro da aréola foi relacionado significativamente com o menor peso da mama $(p<0,0016)$, mostrando que o tamanho da mama teve maior impacto no encontro de invasão do CAM que o diâmetro tumoral. Conclusão: Os resultados desta pesquisa permitiram identificar fatores associados a maior risco de invasão neoplásica areolopapilar.

\section{abstract}

Introduction: The choice for having mastectomy with preservation of the nipple-areolar complex (NAC), with better esthetic results, depends on the abscence of neoplastic invasion of the NAC. Objective: To identify the predictive factors related to neoplastic NAC invasion. Methods: We carried out a retrospective, analytical and clinical-pathologic study comprising 48 mastectomy specimens of breast carcinoma from patients treated at Cancer Institute of Paraíba - Napoleão Laureano Foundation (Hospital de Câncer da Paraíba - Fundação Napoleão Laureano) from April 2007 to January 2008. The mean age was $52.69 \pm$ 10.72. The NAC complex was sectioned separately and perpendicular and parallel cuts were performed from the papilla extending $2 \mathrm{~cm}$ deep into the retroareolar tissue (including collecting ducts, lactiferous sinuses and segmental ducts), followed by transverse cuts toward the deep margin. Results: The frequency of NAC involvement by invasive carcinoma was $14.58 \%$. The following characteristics were associated with a higher risk of NAC involvement ( $\mathrm{p}<0.05$ ): younger patients (mean age $42.3 \pm 4.7$ years), distance between the tumor and the areola/nipple $\leq 2 \mathrm{~cm}$, size of the areola $\leq 3 \mathrm{~cm}$, histological type (invasive ductal carcinoma associated with comedo type ductal carcinoma in situ), status of axillary lymph nodes (mainly with metastasis in 10 or more lymph nodes), presence of lymphovascular and perineural invasion, and inflammatory infiltrate of intra/peritumoral stroma. Smaller areolas correlated significantly with decreased breast weights $(\mathrm{p}<0.0016)$, which demonstrated that the breast size had a greater impact on NAC involvement in comparison with the tumor diameter. Conclusion: The results of this study enabled us to identify factors associated with an increased risk of neoplastic NAC invasion. key words

Breast

Carcinoma

Nipple

\footnotetext{
1. Mestra em patologia; mastologista.

2. Patologista do Hospital de Câncer Napoleão Laureano, João Pessoa-PB.

3. Especialista em Anatomia Patológica pelo American Board of Pathology, EUA; patologista do Hospital de Câncer de Pernambuco.

4. Doutora em Medicina; professora do Departamento de Patologia da Universidade Federal de Pernambuco (UFPE).
} 


\section{Introdução}

O termo cirurgia oncoplástica foi adotado para designar o uso de técnicas de cirurgia plástica em cirurgias de câncer de mama. O cuidado com o aspecto estético da cirurgia oncológica mamária tem-se mostrado importante no tratamento devido às consequências psicológicas da doença e porque é alto o grau de expectativa quanto a um bom resultado ${ }^{(3)}$.

A mastectomia com preservação de pele já tem sua indicação bem definida na mastologia moderna, porém o desafio pela busca de melhores resultados cosméticos e maiores níveis de satisfação fizeram com que, recentemente, fosse adotada nova modalidade de tratamento cirúrgico para câncer de mama: mastectomia preservadora do complexo areolomamilar (variação da de pele), também chamada de adenectomia mamária terapêutica ${ }^{(2)}$. Para tanto, faz-se necessário garantir a integridade do complexo areolomami$\operatorname{lar}($ CAM), obedecendo aos princípios oncológicos, o que inclui, entre outros cuidados, preservar alguns centímetros de tecido sadio no espaço subareolar ${ }^{(29)}$.

A mastectomia com preservação do CAM é um procedimento oncologicamente seguro, desde que o tumor não esteja envolvendo mamilo ou aréola e que a biópsia intracirúrgica do tecido retroareolar exclua comprometimento neoplásico no ato operatório. Assim, uma avaliação de fatores capazes de predizer o envolvimento neoplásico do CAM em câncer de mama é essencial para assegurar a possibilidade de realização dessa técnica cirúrgica ${ }^{(17)}$.

Os objetivos deste estudo foram determinar a frequência de invasão neoplásica do CAM em pacientes com câncer de mama, tratadas por mastectomia, assim como avaliar o valor preditivo dos principais fatores clínicos e anatomopatológicos capazes de predizer o acometimento do CAM para garantir a viabilidade da mastectomia com sua preservação.

\section{Métodos}

O estudo foi analítico, retrospectivo, com amostra não probabilística constituída por 48 peças de mastectomia unilateral de 48 pacientes do sexo feminino, portadoras de câncer de mama, com idade média de 52,69 $\pm 10,72$ anos, variando entre 34 e 75 anos, submetidas a tratamento cirúrgico no Hospital de Câncer da Paraíba - Fundação Napoleão Laureano. Coleta de dados e exame anatomopatológico das peças cirúrgicas foram realizados no período de abril de 2007 a janeiro de 2008.

Como critérios de inclusão foram adotados diagnóstico de câncer de mama (independente de tipo histológico), mastectomia como tratamento cirúrgico empregado e CAM aparentemente livre de infiltração neoplásica ou outras lesões a exames clínico e radiológico. Foram excluídos os casos em que estava presente qualquer uma das seguintes situações: relato de quimioterapia neoadjuvante, doença de Paget (clinicamente diagnosticada) e alterações suspeitas de envolvimento neoplásico do CAM detectadas clinicamente ou no exame macroscópico da peça cirúrgica, como ulcerações, edema, hiperemia e nódulo.

O exame anatomopatológico das peças de mastectomia e a seleção dos cortes para exame histológico foram realizados de acordo com o roteiro proposto no Manual de Padronização de Laudos Histopatológicos da Sociedade Brasileira de Patologia ${ }^{(1)}$. Para exame do CAM desenvolveuse protocolo especialmente para essa pesquisa (Figura 1): 0 CAM foi seccionado separadamente e incluiu todo o tecido glandular mamário retroareolar, até a base da mama, com margens lateral, medial, superior e inferior equivalentes a toda a circunferência da aréola, até $5 \mathrm{~mm}$ a partir de seu contorno. Os cortes do CAM foram perpendiculares e paralelos, a partir da porção distal do mamilo, e se estenderam a $2 \mathrm{~cm}$ de profundidade do tecido retroareolar, com o

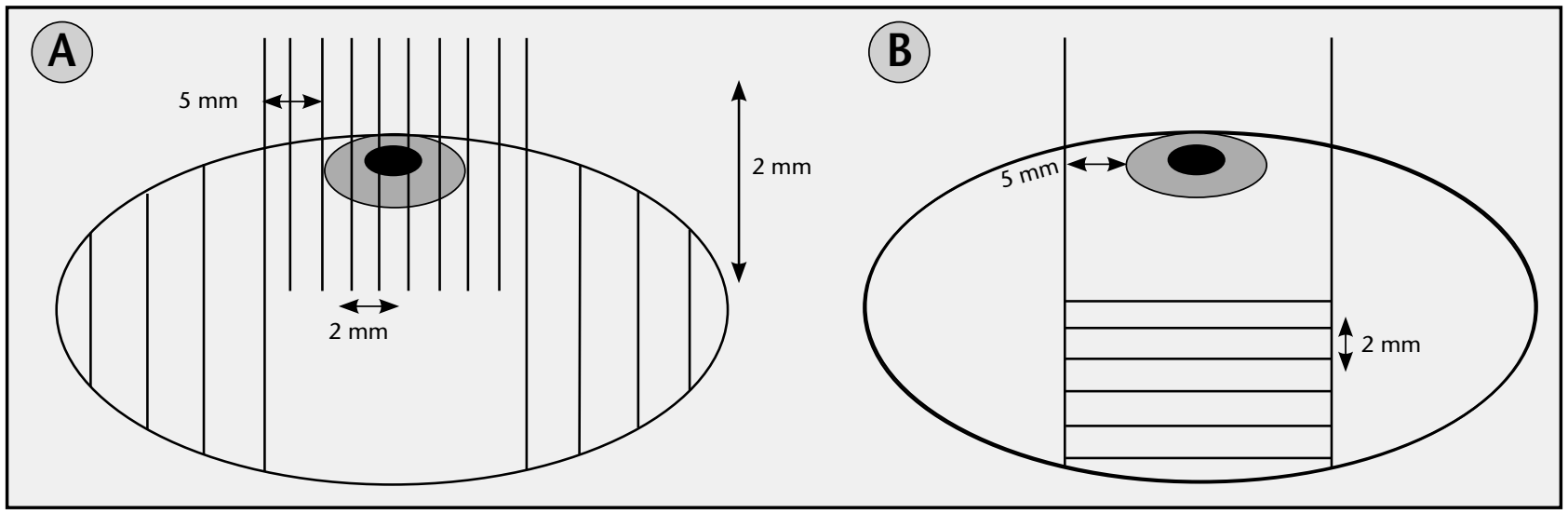

Figura 1 - Diagrama dos cortes macroscópicos da peça cirúrgica. (A) Cortes perpendiculares; (B) cortes transversais 
intuito de incluir os seios lactíferos e ductos segmentares retroareolares e coletores. A partir daí, os cortes passaram a ser transversais até a base da mama. O intervalo entre as secções foi de $2 \mathrm{~mm}$.

As variáveis estudadas abrangeram faixa etária categorizada em intervalos de 10 anos, características tumorais relativas a topografia (categorizada segundo quadrantes mamários), lateralidade da mama acometida, tamanho, tipo histológico, grau histológico, distância do tumor a aréola e papila, maior diâmetro de aréola e papila, status dos linfonodos axilares (positivo ou negativo), número de linfonodos axilares metastáticos, presença de infiltrado inflamatório peritumoral, existência de invasões neoplásicas perineural e angiolinfática e estadiamento pós-cirúrgico (pTNM).

Para avaliar diferenças entre variáveis quantitativas das pacientes com e sem invasão neoplásica do CAM foi usado teste $t$ de Student ou teste de Mann-Whitney, de acordo com normalidade ou homocedacidade dos dados. Variáveis com diferenças significativas foram adotadas na análise multivariada de discriminantes, em que pacientes foram separadas, por eixos adimensionais, em dois grupos (com e sem invasão neoplásica do (AM), estimando-se valores mínimos para cada variável que determinariam de qual grupo um indivíduo poderia fazer parte. Foi determinada a razão de chance $(\mathrm{RC})$ dessas variáveis para estimar o grau de aumento ou diminuição das chances de ter envolvimento neoplásico do CAM.

As variáveis que apresentaram $\mathrm{RC}>1$, indicando que o caráter avaliado é fator de risco para comprometimento neoplásico do CAM, foram agrupadas em análise multivariada de regressão linear logística múltipla para observar a interação das variáveis e estimar as chances de ter CAM positivo. Todas as análises utilizadas tiveram nível de significância de $5 \%$.

Para avaliar a influência da localização dos tumores sobre o aparecimento de neoplasia no CAM, foi realizado o teste Qui-quadrado, com nível de significância de $1 \%$.

\section{Resultados}

Entre as 48 amostras estudadas, houve invasão neoplásica do CAM em sete casos (Figura 2), com frequência igual a $14,58 \%$ (Figura 3), acometendo mulheres com idade média de $42,3 \pm 4,7$ anos e variação de 34 a 49 anos, enquanto 41 pacientes $(85,42 \%)$ sem invasão neoplásica do CAM tinham idade média de $52,7 \pm 10,7$ anos com variação entre 33 a 75 anos.

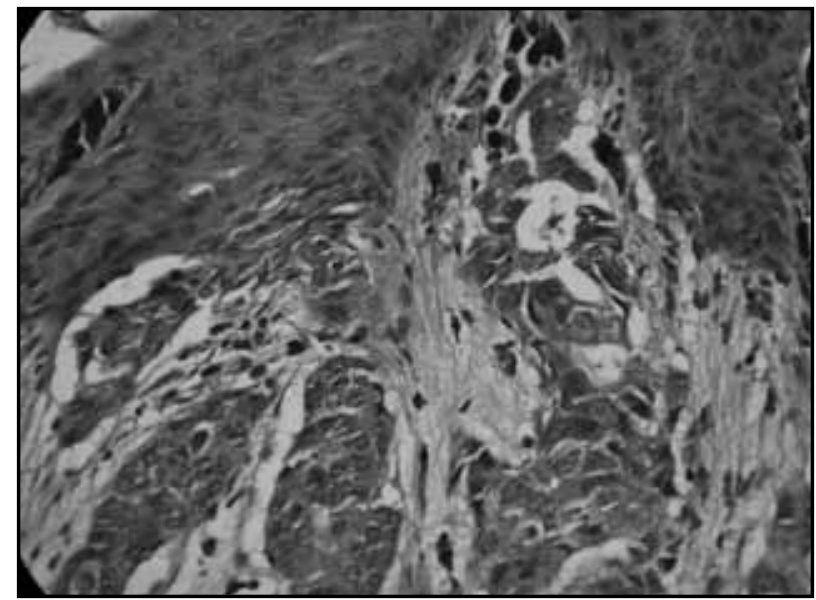

Figura 2 - Invasão do derma da papila por carcinoma ductal invasivo (HE, 400x) $H E$ : hematoxilina e eosina.

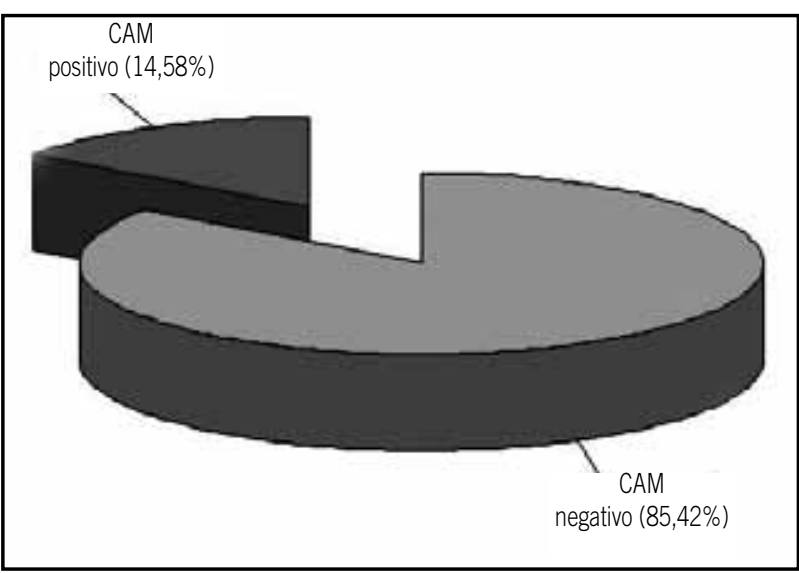

Figura 3 - Percentual das 48 amostras estudadas com presença (CAM positivo) e ausência (CAM negativo) de invasão neoplásica do CAM CAM: complexo areolomamilar.

Ao comparar casos com comprometimento neoplásico do CAM àqueles que não apresentavam essa neoplasia, observaram-se comprometimento neoplásico mais frequente em mama esquerda $(57,1 \%$ contra $63,4 \%$ em mama direita do grupo com CAM livre de neoplasia) e predomínio de topografia mamária equivalente à junção dos quadrantes externos $(28,6 \%)$ e superiores $(28,6 \%)$, diferente do grupo sem invasão neoplásica do CAM, que teve o quadrante superior externo mais acometido $(31,7 \%)$. Constatou-se também que o comprometimento neoplásico do CAM foi mais presente em mamas de volume médio de $500 \mathrm{~g}$, enquanto o daquelas sem acometimento do CAM igualou-se a $1.000 \mathrm{~g}$.

O tipo histológico mais frequente foi o carcinoma ductal invasivo (CDI) puro, que ocorreu em 24 casos (50\%). Entre os sete com invasão neoplásica do CAM, seis apresentaram - CDI associado a carcinoma ductal in situ (CDIS) padrão comedônico $(85,7 \%)$ e um teve diagnóstico de CDI puro (14,3\%). O grau histológico tumoral mais comum foi o III 
$(60,4 \%)$, apresentado pelo grupo que concentrou maior número de amostras com invasão neoplásica do mamilo $(20,7 \%)$.

O tamanho do tumor nas 48 amostras estudadas variou de 1 a $5 \mathrm{~cm}$ em seu maior diâmetro, com média de $3 \pm$ $0,87 \mathrm{~cm}$. O grupo com tumor $\leq 2 \mathrm{~cm}$ (pT1) concentrou maior percentual de casos com invasão neoplásica do CAM $(17,6 \%)$.

Observou-se que quanto maior o número de linfonodos axilares metastáticos, maior o percentual de acometimento neoplásico do CAM, predominando nos casos de metástases com 10 ou mais linfonodos envolvidos (60\%). Em relação à distância do tumor a aréola (DA) e mamilo (DM), as medidas foram categorizadas em intervalos de $1 \mathrm{~cm}$, observando-se maior número de amostras com envolvimento neoplásico do CAM quando a distância foi $\leq 1 \mathrm{~cm}$ tanto para aréola (30\%) quanto para mamilo $(66,7 \%)$.

Quanto ao tamanho da aréola, houve variação de 0,5 a $5,5 \mathrm{~cm}$, com média de $3,4 \pm 1 \mathrm{~cm}$, e o grupo com medidas entre 1,1 e $2 \mathrm{~cm}$ apresentou maior percentual (60\%) de casos com envolvimento neoplásico do CAM. Já o tamanho do mamilo variou de 0,8 a 1,6 cm, com média de 1,3 $\pm 0,5 \mathrm{~cm}$.

Entre as variáveis quantitativas, idade, número de linfonodos axilares metastáticos, tamanho da aréola, DA e DM apresentaram significância estatística $(p<0,05)$. Quanto menor a idade, o tamanho da aréola e a DM/DA e maior o número de linfonodos axilares metastáticos, maior a probabilidade de envolvimento neoplásico do CAM (Tabela 1).

Em relação aos achados histológicos (variáveis dicotômicas), na maioria dos casos com CAM envolvido por células neoplásicas, estiveram presentes infiltrado inflamatório peritumoral e invasões neoplásicas perineural e angiolinfática.
Utilizando-se as equações dos discriminantes de Fisher, observou-se que, na interação dos fatores, o número de linfonodos axilares metastáticos mostrou maior influência estatística. As variáveis dicotômicas, analisadas por meio do teste de RC, que apresentaram significância estatística foram invasões neoplásicas angiolinfática e perineural, infiltrado inflamatório peritumoral e presença de CDIS comedônico associado a CDI. Segundo a RC, a variável individual com maior significância estatística foi a invasão neoplásica perineural, que aumentou em 12 vezes a chance de invasão neoplásica do CAM (Tabela 2).

Quando associadas, as variáveis dicotômicas aumentaram a chance de invasão neoplásica do CAM, conforme regressão linear logística múltipla, mostrando que o fator de maior influência foi a associação de CDIS comedônico $(p<0,01)$.

Constatou-se que quanto menor o peso da mama, menor o tamanho da aréola e maior o acometimento neoplásico do CAM.

As variáveis preditivas de envolvimento neoplásico do CAM foram idade mais jovem (média das amostras sem e com envolvimento neoplásico do CAM $=52,7 \pm 10,7$ anos e $42,3 \pm 4,7$ anos, respectivamente), DM e DA $(\leq 2 \mathrm{~cm})$, tamanho da aréola $(\leq 3 \mathrm{~cm})$, tipo histológico $C D I+C D I S$, número de linfonodos axilares metastáticos $(\geq 10)$, presença de infiltrado inflamatório peritumoral, existência de invasões neoplásicas angiolinfática e perineural (Tabelas 1 e 2).

\section{Discussão}

O envolvimento neoplásico do CAM é definido como a presença de CDIS, CDI ou permeação neoplásica da epiderme (doença de Paget) na papila e/ou no tecido subareolar

\section{Tabela 1 Variáveis analisadas e sua correlação com envolvimento neoplásico do CAM}

\begin{tabular}{lcc}
\hline Variáveis & Presença de invasão neoplásica & Ausência de invasão neoplásica \\
Tamanho do tumor $(\mathrm{cm})$ & 2,6 & 2,5 \\
Distância do tumor à aréola $(\mathrm{cm})$ & $1,8^{*}$ & $3,4^{*}$ \\
Distância do tumor ao mamilo $(\mathrm{cm})$ & $2,3^{*}$ & $4,7^{*}$ \\
Idade & $42^{*}$ & $53^{*}$ \\
Tamanho do mamilo (cm) & 1,3 & 1,3 \\
Tamanho da aréola $(\mathrm{cm})$ & $2,5^{*}$ & $3,8^{*}$ \\
Grau histológico & 3 & 3 \\
Linfonodos axilares metastáticos & $9,5^{*}$ & $2^{*}$
\end{tabular}

Departamento de Anatomia Patológica do Hospital de Câncer Napoleão Laureano (João Pessoa-PB). Período: abril/2007 a janeiro/2008 Teste de Mann-Withney * $\mathrm{p}<0,05$

CAM: complexo areolomamilar. 
Tabela 2 RC aplicada às variáveis dicotômicas

\begin{tabular}{lcc}
\hline Variável & RC individual (IC 95\%) & RC associada (IC 95\%) \\
Infiltrado inflamatório peritumoral & $4,1(0,6-32,1)$ & $30,8(1,8-1571,4)$ \\
Invasão neoplásica perineural & $12,9(1,3-615)$ & $106(15,4-378,1)$ \\
Invasão neoplásica angiolinfática & $3,8(0,4-187,5)$ & $4.298(40,58-5795,41)$ \\
CDIS comedônico & $7,2(1,3-314,9)$ & $5,6(1,79-17,9)$ \\
\hline
\end{tabular}

Departamento de Anatomia Patológica do Hospital de Câncer Napoleão Laureano (João Pessoa-PB). Período: abril/2007 a janeiro/2008

Regressão linear logística múltipla

$R C$ : razão de chance; IC: intervalo de confiança; CDIS: carcinoma ductal in situ.

em profundidade de até $1 \mathrm{~cm}^{(4)}$. Neste estudo, a invasão neoplásica do CAM foi encontrada em sete das 48 amostras estudadas (14,58\%).

Cense et $a l^{(7)}$, relatando experiência internacional de vários autores, observaram que a porcentagem de envolvimento neoplásico do CAM varia de $0 \%$ a 58\%. Quinn e Barlow ${ }^{(21)}$, estudando 45 espécimes de mastectomia, encontraram $25 \%$ de envolvimento neoplásico do CAM, enquanto na série de 414 casos de Raafat e Hindawy ${ }^{(22)}, 26$ casos (6,3\%).

As diferenças nas taxas de envolvimento neoplásico do CAM são discrepantes devido à falta de padronização na análise histológica e nos critérios de exclusão. Quanto maior for o número de cortes histológicos, envolvendo maior número de ductos mamários a ser analisado, maior será a probabilidade de encontrar acometimento neoplásico ${ }^{(1)}$. Há divergência também na profundidade do CAM que é estudada.

Neste trabalho, o CDI foi o tipo histológico mais frequente (50\%) e sua associação a CDIS comedônico apresentou forte impacto sobre o acometimento neoplásico do CAM (85,7\%). Esses dados corroboram os achados de Morimoto et al. ${ }^{(16)}$ e Vlajcic et al. ${ }^{(27)}$, que associaram a maior chance de comprometimento do CAM com carcinoma invasivo acompanhado de CDIS extenso.

O CDIS de padrão comedônico está relacionado com maior diâmetro tumoral, quando comparado às formas não comedônicas de baixo grau, como também a incidência de invasão oculta é muito mais comum em CDIS tipo comedo, sobretudo naqueles com diâmetro maior que $2,5 \mathrm{~cm}$. Dessa forma, constitui importante fator de recorrência local e metástase axilar $(5,9,11,18,20,23,25)$.

O índice prognóstico de Van Nuys quantifica preditores significativos de recorrência local em CDIS, e a presença do padrão comedo constitui fator relevante, como também o são tamanho da neoplasia in situ e status das margens cirúrgicas ${ }^{(25)}$.

Em geral, o CDIS padrão comedônico apresenta alto índice proliferativo, na avaliação imuno-histoquímica, pelo anticorpo Ki-67 e imunoexpressão do HER2 em escore 3+.
Esse tipo histológico geralmente é negativo para receptores hormonais. Seu crescimento dentro da árvore ductal é contínuo em $90 \%$ dos casos e multifocal em $10 \%$. Calcificações distróficas estão frequentemente presentes nos lumens dos ductos comprometidos por CDIS de padrão arquitetural comedônico e são importantes para deteç̧ão dessa neoplasia à mamografia.

Nesta pesquisa, a associação de neoplasia invasiva com CDIS padrão comedo representou relevante fator preditivo de invasão do CAM, o que se explica por sua relação com maior agressividade local e padrão de crescimento, no qual células neoplásicas crescem dentro de uma área da árvore ductal de modo contínuo e unicêntrico, podendo chegar até o ducto lactífero correspondente no CAM. A presença de CDIS tipo comedo aumentou em sete vezes a chance de invasão neoplásica do CAM $(R C=7,2)$.

Recentemente, Brachtel et al. ${ }^{(6)}$ verificaram associação de envolvimento da papila com imunoexpressão (escore 3+) ou amplificação do HER2 e alto grau histológico dos carcinomas invasivo e in situ, o que corrobora nossos achados de associação com carcinomas invasivo e in situ de alto grau. Esses mesmos autores relatam, ainda, relação entre tamanho tumoral, DM e metástase linfonodal com achado de $21 \%$ de envolvimento do CAM em mastectomias terapêuticas.

Neste estudo, não foi encontrada diferença significativa quanto ao tamanho do tumor, divergindo da maioria dos autores que apresentaram importante correlação entre invasão do CAM e tumores maiores que 3 a $4 \mathrm{~cm}^{(7,12,22)}$. No entanto, alguns autores também não encontraram associação entre o tamanho do tumor e o envolvimento neoplásico do $\mathrm{CAM}^{(10)}$.

Na série de Laronga et al.(13), não houve diferença significativa entre acometimento neoplásico do CAM e fatores como tamanho do tumor, grau histológico, tipo histológico e status dos receptores hormonais. Saliente-se ainda que, mesmo apresentando pequenas dimensões, o tumor pode possuir alta capacidade proliferativa e potencial metastático, 
assim como outros fatores devem ser considerados antes de determinar o significado prognóstico de tumor de tamanho pequeno. Essas afirmações foram observadas neste trabalho, uma vez que tumores de menor dimensão apresentaram maior número de linfonodos axilares metastáticos.

Associação do diâmetro areolar $\leq 2 \mathrm{~cm}$ com invasão neoplásica do CAM reflete menor tamanho da mama como fator preditor, contraindicando cirurgia conservadora. Além disso, Schnitt et al.(24) mostraram, ao investigarem aréolas de peças de mastectomia, que ductos mamários são componentes normais da derme areolar e, portanto, seu envolvimento neoplásico pode ser mais evidente em aréolas de menor tamanho pela própria distribuição anatômica ductal em área superficial menor.

A distância mínima entre tumor e mamilo é considerada importante fator de risco para o envolvimento neoplásico do $\mathrm{CAM}^{(7,12)}$. Com base em nossos achados, a distância entre tumor e CAM teve influência significativa na infiltração neoplásica, pois quanto menor essa distância, maior a possibilidade de acometimento areolomamilar, resultados esses semelhantes aos verificados por muitos

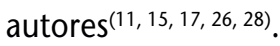

O número de linfonodos axilares comprometidos tem sido apontado como importante fator preditivo de envolvimento neoplásico do $\mathrm{CAM}^{(22)}$. A literatura mostra correlação entre metástases em linfonodos axilares e envolvimento neoplásico do CAM em percentual que varia de $11 \%$ a 54\% dos casos, enquanto nas pacientes sem metástase axilar, de $3,5 \%$ a $27 \%{ }^{(7)}$. Nesta pesquisa, os linfonodos axilares acometidos apresentaram forte influência na invasão neoplásica do CAM, tanto como fator isolado quanto na análise multivariada, na qual mostrou ser o fator de maior relevância, sobretudo quando o número de linfonodos acometidos era maior ou igual a 10 (pN3), mostrando alto valor preditivo, mesmo associado a tumores de menor tamanho.

A associação entre acometimento do CAM e permeação neoplásica angiolinfática corroborou a relação entre status axilar e invasão neoplásica do CAM, pois sua presença indica maior possibilidade de metástases para linfonodos axilares, também verificada por outros autores ${ }^{(6)}$.

A presença de infiltrado inflamatório (mononuclear) tem sido relacionada com graus histológicos altos e, portanto, tumores com maior potencial de recidiva local ${ }^{(19)}$. No entanto, o significado prognóstico dessa associação é controverso. Alguns estudos referiram efeito adverso de infiltração mononuclear proeminente no resultado clínico, enquanto outros pesquisadores observaram efeito benéfico ${ }^{(24)}$. Em nossa série, apesar do infiltrado inflamatório peritumoral ter-se mostrado importante fator isolado, na análise de regressão linear sua influência estatística foi menor que a dos demais fatores.

A invasão angiolinfática é fator prognóstico importante e independente, que está relacionada com recorrência do câncer de mama ${ }^{(24)}$. Na série de Rosen et al.(. ${ }^{(23)}$, durante 20 anos de acompanhamento, houve correlação entre invasão angiolinfática e recidiva local. A taxa de recidiva local para pacientes com estádio I do câncer de mama e invasão angiolinfática presente foi de $38 \%$ vs. $22 \%$ para pacientes com ausência dessas características.

Na série de Vlajcic et al. ${ }^{(27)}$, houve diferença significativa quanto a DM, tamanho do tumor, comprometimento neoplásico axilar e invasão angiolinfática. Nesta pesquisa, a invasão angiolinfática mostrou ser fator preditivo de envolvimento neoplásico do CAM, pois aumentou o risco em três vezes, quando analisado individualmente, e apresentou significância ainda maior quando associado. Para Vlajcic et al. ${ }^{(27)}$, as indicações clínicas para mastectomia com preservação do CAM são tumores $\leq 2,5 \mathrm{~cm}, \mathrm{DM}>4 \mathrm{~cm}$, status linfonodal axilar negativo e ausência de invasão angiolinfática. A existência de invasão neoplásica perineural, nesta série, também pareceu atuar como preditor de infiltração neoplásica do CAM.

\section{Conclusão}

Na ausência de localização tumoral retroareolar e de alterações clínicas ou macroscópicas suspeitas de envolvimento neoplásico do CAM, os seguintes achados associaram-se significativamente a maior frequência de envolvimento do CAM: idade $<50$ anos, diâmetro da aréola $\leq 3 \mathrm{~cm}$ (que se relacionou com menor peso da mama), DA/DM $\leq 2 \mathrm{~cm}$, ocorrência de CDIS padrão comedônico associado a componente invasivo, número de linfonodos acometidos por metástases $\geq 10$, invasões angiolinfática e perineural e infiltração inflamatória dos estromas peritumoral e intratumoral. Vários desses parâmetros, bem como outros considerados preditivos do envolvimento do CAM por outros autores, podem ser frequentemente determinados antes da mastectomia, podendo ser utilizados na seleção das pacientes candidatas à mastectomia com preservação do CAM. São eles: idade, diâmetro da aréola, tamanho da mama, localização e tamanho do tumor, DA/DM (por exames de imagem), ocorrência de CDIS padrão comedônico, alto grau histológico e imunoexpressão escore 3+ para HER-2, tanto do componente invasivo como do in situ (por biópsia pré-operatória). Além disso, é recomendável biópsia intracirúrgica do tecido retroareolar ou subareolar. 


\section{Referências}

1. ABREU E LIMA, M. C. C. et al. Lesões benignas não neoplásicas e neoplasias da mama. In: BACCHI, C. E.; ALMEIDA, P. C. C.; FRANCO, M. Manual de padronização de laudos histopatológicos. Sociedade Brasileira de Patologia. 3. ed. São Paulo: Reichmann \& Autores Editores, 2005. cap. 25. p. 245-81.

2. AFIFI, R. Y.; EL-HINDAWY, A. Analysis of nipple-areolar complex involvement with mastectomy: can the nipple be preserved in Egyptian patients receiving skin-sparing mastectomy? Breast J, v. 10, n. 6, p. 543-5, 2004.

3. AMANTI, C. et al. Total periareolar approach in breastconserving surgery. Tumori, v. 89, n. 4, p. 169-72, 2003.

4. ANDERSEN, J. A.; PALLESEN, R. M. Spread to the nipple and areola in carcinoma of the breast. Ann Surg, v. 189, n. 3, p. 367-72, 1979.

5. AZZOPARDI, J. G. Problems in breast pathology. Philadelphia: WB Saunders, 1983.

6. BRACHTEL, E. F. et al. Occult nipple involvement in breast cancer: clinicopathologic findings in 316 consecutive mastectomy specimens. J Clin Oncol, v. 27, p. 4948-54, 2009.

7. CENSE, H. A. et al. Nipple-sparing mastectomy in breast cancer: a viable option? Eur J Surg Oncol, v. 27, n. 6, p. 521-6, 2001.

8. FISHER, B.; ANDERSON, S.; BRYANT, J. Twenty-year follow up of a randomized trial comparing total mastectomy, lumpectomy and lumpectomy plus irradiation for the treatment of invasive breast cancer. N Engl J Med, v. 347, n. 16, p. 1233-41, 2002.

9. HWANG, E. S.; ESSERMAN, L. J. Tratamento do carcinoma ductal in situ. In: JATOI, I. Clínicas cirúrgicas da América do Norte. Cond Cancer Mama, v. 79, n. 5, p. 929-51, 1999.

10. KISSIN, M. W.; KLARK, A. E. Nipple preservation during mastectomy. Br J Surg, v. 4, p. 58-61, 1987.

11. LAGIOS, M. D. et al. A guide to the frequency of nipple involvement in breast cancer. A study of 149 consecutive mastectomies using a serial subgross and correlated radiographic technique. Am J Surg, v. 138, p. 135-41, 1979.

12. LAMBERT, P.A.; KOLM, P.; PERRY, R. R. Parameters that predict nipple involvement in breast cancer. J Am Coll Surg, v. 191, n. 4, p. 354-9, 2000.

13. LARONGA, C. et al. The incidence of occult nipple-areola complex involvement in breast cancer patients receiving a skin-sparing mastectomy. Ann Surg Oncol, v. 6, n. 6, p. 609-13, 1999.

14. LENNINGTON, W. J. et al. Ductal carcinoma in situ of the breast: heterogeneity of individuals lesions. Cancer, v. 73, p. 118, 1994.

15. LUTTGES, J.; KALBFLEISC, H.; PRINZ, P. Nipple involvement and multicentricity in breast cancer. $A$ study on whole organ sections. J Cancer Res Clin Oncol, v. 113, p. 481-7, 1987.

16. MORIMOTO, T. et al. Preservation of the nipple and areola for breast cancer-histopathological study on cancerous involvement of the nipple and areola. Cancer, v. 15, n. 55 , p. 2459-63, 1985.
17. MORKBEL, R.; MORKBEL, K. Is it safe preserve the nipple areola complex during skin-sparing mastectomy for breast cancer? Int J Fertil Womens Med, v. 51, n. 5, p. 230-2, 2006.

18. MORROW, M.; SCHNITT, S. J.; HARRIS, J. R. Carcinoma ductal in situ. Carcinoma microinvasivo. In: HARRIS, J. R. et al. Doenças da mama. 2. ed. Rio de Janeiro: Medsi, 2002. cap 27. p. 461-82.

19. NIXON, A. J.; SCHNITT, S. J.; GELMAN, R. Relationship of tumor grade to other pathologic features and to treatment outcome of patients with early stage breast carcinoma treated with breast conserving therapy. Cancer, v. 78, p. 1426, 1996.

20. PATCHEFSKY, A. S.; SCHWARTZ, G. F.; FINKELSTEIN, S. I. Heterogeneity of intraductal carcinoma of the breast. Cancer, v. 63, p. 731, 1989.

21. QUINN, R. H.; BARLOW, J. F. Involvement of the nipple and areola by carcinoma of the breast. Arch Surg, v. 116, n. 9, p. 1139-40, 1981.

22. RAAFAT, Y. A.; HINDAWY, A. E. Analysis of nipple-areolar complex involvement with mastectomy: can the nipple be preserved in Egyptian patients receiving skin-sparing mastectomy? The Breast J, v. 10, n. 6, p. 543-5, 2004.

23. ROSEN, P. P.; GROSHEN, S.; SAIGO, P. E. Pathological prognostic factors in stage I (T1NOMO) and stage II (T1N1M0) breast carcinoma: a study of 644 pacients with median follow-up of 18 years. J Clin Oncol, v. 7, p. 1239, 1989.

24. SCHNITT, S. J.; GUIDI, A. J. Patologia do câncer de mama invasivo. In: HARRIS, J. R. et al. Doenças da mama. 2. ed. Rio de Janeiro: Medsi, 2002. cap 27. p. 509-64.

25. SILVERSTEIN, M. J.; WAISMAN, J. R.; GAMAGAMI, P. Intraductal carcinoma of the breast (208 cases): clinical factors influencing treatment choice. Cancer, v. 66, p. 102, 1990.

26. VERMA, G. R.; KUMAR, A.; JOSHI, K. Nipple involvement in peripheral breast carcinoma: a prospective study. Indian J Cancer, v. 34, p. 1-5, 1997.

27. VLAJCIC, Z. et al. Nipple-areola complex preservation: predictive factors of neoplastic nipple-areola complex invasion. Ann Plast Surg, v. 55, n. 3, p. 240-4, 2005.

28. VYAS, J. J.; CHINOY, R. F.; VAIDYA, J. S. Prediction of nipple and areola involvement in breast cancer. Eur $J$ Surg Oncol, v. 24, p. 15-6, 1998.

29. WIJAYANAYAGAM, A. et al. Optimizing the total skinsparing mastectomy. Arch Surg, v. 143, n. 1, p. 38-45, 2008. 